\title{
Immunodeficiency-Related Malignant Neoplasm
}

National Cancer Institute

\section{Source}

National Cancer Institute. Immunodeficiency-Related Malignant Neoplasm. NCI

Thesaurus. Code C27770.

Malignant neoplasm occurring in immunodeficient patients. 